\title{
Explicit Model Predictive Control Approach for Low-Thrust Spacecraft Proximity Operations
}

\author{
Mirko Leomanni* \\ Università di Siena, 53100 Siena, Italy \\ and \\ Eric Rogers $₫$ and Stephen B. Gabriel ${ }^{ \pm}$ \\ University of Southampton, Southampton, England SO17 1BJ, United Kingdom
}

DOI: $\underline{10.2514 / 1 . G 000477}$

\begin{abstract}
The key role of autonomous systems in future space missions has made model predictive control a very attractive guidance and control technique. However, the capability of low-power spacecraft processors to handle the real-time computational load of this technique still needs to be fully established, especially for complex control problems. This paper introduces a method to improve the computational efficiency of model predictive control when applied to the problem of autonomous rendezvous and proximity maneuvering using low-thrust propulsion. To ensure safe trajectories in this scenario, a long control horizon is required and the control problem must be solved at a relatively fast sampling rate. The proposed design addresses such requirements by parameterizing the thrust profile with a set of Laguerre functions. In this setting, the number of control variables can be made significantly smaller than the length of the control horizon, as opposed to standard design methods. By exploiting this property, in combination with multiparametric programming techniques, an explicit control law is derived that is suitable for real-time implementation on simple hardware. The performance of this approach is demonstrated on a small spacecraft mission and compared with that of other control techniques.
\end{abstract}

\section{Introduction}

$\mathbf{T}$ HE development of guidance and control techniques for spacecraft formation flying is the subject of significant research efforts, due to the key role of such problems in many present and future space missions. Examples include technology demonstrators like PRISMA [1] and PROBA-3 [2], the space interferometer DARWIN [3], the Mars sample return scientific mission [4], and onorbit servicing projects such as the Automated Transfer Vehicle [ㅁ] or the orbital life extension vehicle SMART-OLEV [6].

Of particular interest in this field is the optimization of low-thrust formation flying trajectories, motivated by the application of miniaturized or high-efficiency propulsion technologies [7-10]. When two or more spacecraft in a formation are required to operate in close proximity, these trajectories must be safe with respect to collisions and other possible anomalies [11]. This generally leads to complex trajectory optimization problems that are subject to both thrust magnitude and path constraints. Because of the increasing level of autonomy of future space applications, it is critical to compute the solution to these problems in real time and to design a control system tracking the resulting trajectories [12]. To this purpose, efficient guidance and control algorithms must be devised. More specifically, this paper tackles the problem of developing an optimal guidance and control scheme for autonomous rendezvous and proximity maneuvering using low-thrust propulsion, in the presence of collision avoidance, thruster plume impingement, and line-of-sight (LoS) constraints.

A wide variety of open-loop guidance techniques have been proposed in the literature for the design of low-thrust rendezvous trajectories, based on either direct or indirect optimization methods [13-15]. These techniques are known to provide accurate numerical

Received 29 December 2013; revision received 9 May 2014; accepted for publication 27 May 2014; published online 28 August 2014. Copyright (C) 2014 by the American Institute of Aeronautics and Astronautics, Inc. All rights reserved. Copies of this paper may be made for personal or internal use, on condition that the copier pay the $\$ 10.00$ per-copy fee to the Copyright Clearance Center, Inc., 222 Rosewood Drive, Danvers, MA 01923; include the code 1533-3884/14 and \$10.00 in correspondence with the CCC.

*Ph.D. Student, Department of Information Engineering and Mathematical Sciences; leomanni@dii.unisi.it.

${ }^{\dagger}$ Professor, Electronics and Computer Science; etar@ecs.soton.ac.uk.

†Professor, Electronics and Computer Science; sbg2@ soton.ac.uk. solutions, but they cannot cope with the high degree of autonomy required by applications in which disturbance rejection and robustness with respect to perturbations are of primary concern. To circumvent this issue, feedback guidance and control algorithms, with the ability to systematically handle thrust magnitude and path constraints, are commonly used. In particular, model predictive control (MPC), based on computing the optimal control sequence over a finite number of future sampling instances under a receding horizon strategy, is becoming increasingly attractive [16-19]. In lowthrust problems, however, a long control horizon is needed to guarantee adequate performance, due to the limited control authority provided by the actuators. During close proximity operations, this is coupled with the requirement to use a small discretization step to avoid the violation of path constraints between discrete time samples. In such cases, the main drawback of MPC is the requirement to solve a trajectory optimization problem with a large number of decision variables at each time sample, which may make this method too computationally intensive to be implemented on-line on low-power spacecraft processors [20].

A possible way of enhancing MPC to overcome this last difficulty is to parameterize the control sequence with a set of Laguerre functions, where the poles of these functions are used to reflect the time scale of the control system, see [21]. In this setting, which belongs to the family of direct optimization methods, the number of decision variables can be made significantly smaller than the length of the control horizon, whereas path constraints can still be enforced over a sufficiently fine discretization grid.

Another important factor, which may prevent the implementation of the MPC design methods discussed so far, is the requirement to embed a control solver with guaranteed runtime onboard the spacecraft. This requirement can be avoided by solving the control problem explicitly, i.e., by finding a feedback control law offline defined on a partition of the state space [22]. In the standard MPC framework, however, this is generally feasible only for low-dimensional problems, due to the exponential growth of the number of regions in the partition with the length of the control sequence [23]. An alternative approach, based on the explicit solution of a quadratically constrained linear quadratic regulator (LQR) problem, has been recently proposed in [24] for a rendezvous problem with thrust constraints, which confirms the need for computationally efficient feedback control methods specifically tailored to the considered application area. 
The contribution of this paper is twofold. First, a low-complexity MPC scheme is developed for the low-thrust rendezvous and proximity maneuvering problem. In the derivation of the control algorithm, the trajectory optimization problem is reformulated by parameterizing the control sequence by a set of Laguerre functions, which allows a long control horizon to be realized without using a large number of decision variables. Then, an explicit control law is derived by exploiting this new algorithm in combination with multiparametric programming techniques. Such design provides a tradeoff between feasibility and performance of the guidance and control system. Because online optimization is not required, the novel control law is especially suitable for real-time implementation onboard small spacecraft with limited computational capabilities. A detailed simulation-based assessment of the performance achievable under this design is given for an example cubesat mission using a miniaturized electric propulsion system, in comparison to standard MPC and LQR techniques.

The paper is organized as follows. In Sec. II, proximity operations, including terminal rendezvous and docking, are briefly described. Section III then details the main features of the control problem and presents the novel control law and Sec. IV illustrates the formation flying model used to validate the proposed approach. The performance of the control law is evaluated through numerical simulations in Sec. $\underline{\mathrm{V}}$. Section VI gives some concluding remarks.

\section{Problem Setting}

The considered problem is that of autonomous rendezvous and proximity operations between two spacecraft in a leader-follower formation, where the attitude of both spacecraft is actively controlled and the leader is not maneuvering. Based on relative position measurements from differential GPS and optical sensors, the follower spacecraft is required to maintain visual contact and dock with the leader, using low-thrust propulsion. The control objective is to minimize a combination of the fuel expenditure and the time of flight of the maneuver [25], subject to the following requirements to ensure safe trajectories [11] :

1) collision avoidance: the spacecraft must not collide with each other;

2) LoS: the relative motion must be confined within a certain region of the state space (a cone) to maintain visual contact;

3) plume impingement: the magnitude and/or amount of thruster firings directed toward the leader must be minimized during the final phase of the approach.

In addition, thrust magnitude and direction constraints must be taken into account in the control problem.

In this paper, vectors are denoted by boldface italic symbols where $\boldsymbol{1}$ and $\boldsymbol{0}$ denote vectors whose components are all equal to 1 or 0 , respectively - and matrices are denoted by boldface symbols. The identity matrix is denoted by $\mathbf{I}$ and the symbol $\mathbf{0}$ denotes the null matrix of compatible dimensions. The symbol $\bigoplus^{n} \mathbf{A}$ denotes a block diagonal matrix with $n$ diagonal blocks, each equal to $\mathbf{A}$ and the norm of a vector is denoted by $\|\cdot\|_{n}$, where the $\infty, 1$, and 2 norms are used. Moreover, the relative motion of the formation is expressed in a rotating local-vertical-local-horizontal (LVLH) frame centered at the leader spacecraft's center of mass. The $Z$ axis points toward the Earth's center of mass, the $Y$ axis is aligned with the negative orbit normal, and the $X$ axis completes an orthogonal right-handed coordinate system, as illustrated in Fig. 1 . In a circular orbit, the $X$ axis is aligned with the spacecraft's velocity vector. The $X, Y$, and $Z$ directions are referred to as the along-track, cross-track, and radial directions, respectively. The $X-Y$ and the $X-Z$ planes are referred as the horizontal plane and the in-plane, respectively, and the relative position vector is denoted by

$$
\delta \boldsymbol{r}=\left[\begin{array}{lll}
x & y & z
\end{array}\right]^{T}
$$

where $x, y$, and $z$ are the along-track, cross-track, and radial components, respectively.

The following assumptions are made on the configuration of the formation: 1) the leader orbit is nearly circular, 2) the distance

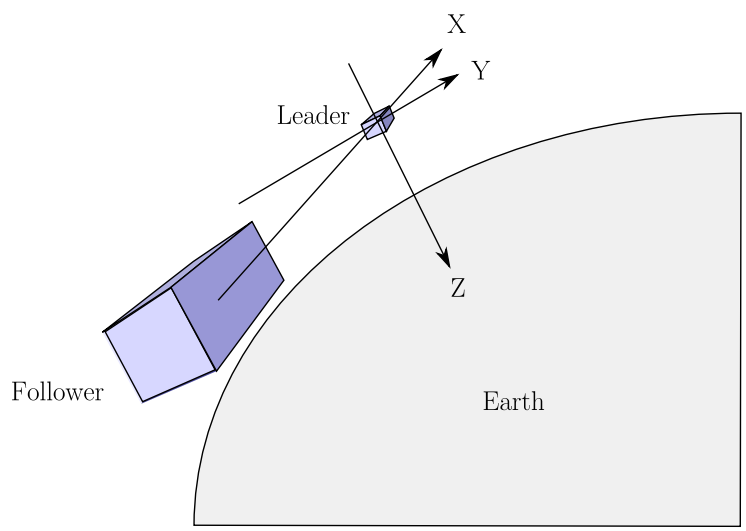

Fig. 1 LVLH reference frame.

between the two spacecraft is small compared to the orbit radius, and 3) differential perturbations are negligible. Under these assumptions, the relative motion dynamics are well approximated by the linearized Hill-Clohessy-Wiltshire (HCW) equations [26]

$$
\ddot{x}=2 \omega \dot{z}+\frac{u_{1}}{m} \quad \ddot{y}=-\omega^{2} y+\frac{u_{2}}{m} \quad \ddot{z}=3 \omega^{2} z-2 \omega \dot{x}+\frac{u_{3}}{m}
$$

where $u_{1}, u_{2}$, and $u_{3}$ are the control forces of the follower expressed in the LVLH frame, $m$ is the mass of the spacecraft, and $\omega$ is the LVLH rate.

Moreover, it is assumed that: 4) both the leader and follower spacecraft are three-axis stabilized to maintain the LVLH attitude, 5) the docking port is located behind the leader, and 6) the propulsion system of the follower can produce thrust only in the along-track and cross-track directions. The position of the docking port can be expressed in terms of relative states as

$$
\delta \boldsymbol{r}_{d}=\left[\begin{array}{lll}
x_{d} & 0 & 0
\end{array}\right]^{T}
$$

where $x_{d} \leq 0$ is fixed. Because radial thrust is not available, $u_{3}=0$ in Eq. (2) and the input vector is defined as

$$
\boldsymbol{u}=\left[\begin{array}{ll}
u_{1} & u_{2}
\end{array}\right]^{T}
$$

In this setting, any arbitrary initial state $\delta \boldsymbol{r}\left(t_{0}\right)$ can be steered to $\delta \boldsymbol{r}_{d}$, because the in-plane motion in Eq. (2) is controllable with the single input $u_{1}$ [27]. The tracking error is denoted by

$$
\boldsymbol{x}=\left[\begin{array}{lll}
x_{1} & \ldots & x_{6}
\end{array}\right]^{T}=\left[\begin{array}{ll}
\left(\delta \boldsymbol{r}-\delta \boldsymbol{r}_{d}\right)^{T} & \left(\delta \dot{\boldsymbol{r}}-\delta \dot{\boldsymbol{r}}_{d}\right)^{T}
\end{array}\right]^{T}
$$

where $\delta \boldsymbol{r}_{d}=\boldsymbol{0}$, because $\delta \boldsymbol{r}_{d}$ is fixed according to Eq. (3).

\section{Formation Control}

Let $\mathbb{U}$ be an admissible input set, $\mathbb{X}$ be an admissible subset of the state space defined by path constraints, and $J(\boldsymbol{x}, \boldsymbol{u})$ be a given cost function, defined over the time interval $t \in\left[t_{0}, t_{f}\right]$. In the considered problem, the input set is bounded by the maximum thrust $u_{M}$ that can be delivered by the propulsion system, as

$$
\mathbb{U}=\left\{\boldsymbol{u}:\|\boldsymbol{u}(t)\|_{\infty} \leq u_{M}\right\}
$$

Collision avoidance and LoS requirements can be expressed as the path constraints

$$
\mathbb{X}=\left\{x: x_{1}(t) \leq 0, \sqrt{x_{2}(t)^{2}+x_{3}(t)^{2}} \leq-x_{1}(t) \tan (\theta / 2)\right\}
$$

where $\theta$ is the field of view of the optical sensor onboard the follower spacecraft. For rendezvous and docking of a leader-follower spacecraft pair, a relevant cost function is 


$$
J(\boldsymbol{x}, \boldsymbol{u})=\alpha \int_{t_{0}}^{t_{f}}\|\boldsymbol{u}(t)\|_{1} \mathrm{~d} t+(1-\alpha) \int_{t_{0}}^{t_{f}} 1 \mathrm{~d} t+\beta \int_{t_{0}}^{t_{f}} \epsilon(t) \mathrm{d} t
$$

where the final time $t_{f}$ is free, $\alpha \in[0,1]$ is a relative weight on the fuel consumption (first term) and the maneuver time (second term), and $\beta \geq 0$ is a weight on the function $\epsilon$, which accounts for plume impingement requirements. Assuming that Eq. (7) is satisfied, the thruster plume impingement function can, as justified in [28], be taken as

$$
\epsilon(t)=\left\{\begin{array}{cc}
u_{1}^{-}(t) & \text { if }-x_{1}(t) \leq x_{\epsilon 1},\left|x_{2}(t)\right| \leq x_{\epsilon 2} \text { and }\left|x_{3}(t)\right| \leq x_{\epsilon 3} \\
0 & \text { otherwise }
\end{array}\right.
$$

where $u_{1}^{-}(t)$ is the negative part of the along-track thrust and $x_{\epsilon 1}, x_{\epsilon 2}$, and $x_{\epsilon 3}$ are predefined positive values. Given $\boldsymbol{x}\left(t_{0}\right)$, the formation control problem can be stated as

$$
\begin{aligned}
& \min _{\boldsymbol{u}} J(\boldsymbol{x}, \boldsymbol{u}) \\
& \text { s.t. } \quad(2) \\
& \qquad \boldsymbol{x} \in \mathbb{X}, \quad \boldsymbol{u} \in \mathbb{U} \\
& \boldsymbol{x}\left(t_{f}\right)=\boldsymbol{0}
\end{aligned}
$$

The problem defined by Eq. (10) does not admit a closed-form solution and must be solved numerically. Moreover, because Eq. (10) consists of a nonlinear nonsmooth optimization problem, its online solution onboard spacecraft with limited computational capabilities may not be possible. Instead, a number of suboptimal policies have been considered in the literature [16-19,24,29,30].

MPC is a potential design method for the problem described previously, because it enables constraints to be placed on inputs and outputs and the control law can be explicitly parameterized in feedback form. In linear MPC, the endpoint equality constraint $\boldsymbol{x}\left(t_{f}\right)=\boldsymbol{0}$ is typically replaced by a weight $\mathbf{W}_{f}$ on the terminal state of the system, the set $\mathbb{X}$ is approximated by a polyhedral set $\bar{X}$, and the problem is formulated over a finite horizon $T_{p}=\left(t_{f}-t_{0}\right)$ as

$$
\min _{\boldsymbol{u}} J_{c}(\boldsymbol{x}, \boldsymbol{u})=\left\|\mathbf{W}_{f} \boldsymbol{x}\left(t_{f}\right)\right\|_{n}^{n}+\int_{t_{0}}^{t_{f}}\|\mathbf{W} \boldsymbol{x}(t)\|_{n}^{n}+\|\mathbf{K} \boldsymbol{u}(t)\|_{n}^{n} \mathrm{~d} t
$$

s.t. $\quad(2)$

$$
x \in \overline{\mathbb{X}}, \quad u \in \mathbb{U}
$$

where $\mathbf{W}_{f}, \mathbf{W}$, and $\mathbf{K}$ are square weighting matrices, $\mathbf{K}$ is nonsingular, and a value of $n=1$ or 2 is considered in this paper. Feedback control of the formation is obtained by solving the discretized form of problem Eq. (11) under the receding horizon principle, where the resulting closed-loop system can be shown to be asymptotically stable for a proper tuning of the MPC design parameters, see [31]. To ensure an acceptable computational complexity, the nonconvex plume impingement function (9) is not included in Eq. (11). A possible shortcoming of this approximation is that the control law obtained for $n=1$, which takes into account the fuel consumption, may not be compatible with plume impingement requirements due to the bang-bang structure of the optimizer [32]. Conversely, smooth control trajectories from the solution of the relaxed problem for $n=2$ in Eq. (11) are feasible, because in this case the control magnitude generally vanishes close to the steady state. These considerations, together with the fact that the minimum energy problem has proven to be effective in approximating the minimum fuel problem [33], even when radial thrust is not available [27], motivates the choice of $n=2$ in Eq. (11).

In this work, a low-complexity explicit solution of the MPC problem is sought. It is known that, in the worst case, the number of state-space regions over which an explicit control law is defined grows exponentially with the length of the input sequence [23]. This problem could well arise in the application considered in this paper because the control problem must be defined over a long time horizon to account for the limited control authority and discretized with a relatively small step to avoid the violation of path constraints between discrete time samples. This issue is addressed by parameterizing the input sequence with a set of Laguerre functions, see $[\underline{21}, \underline{34}]$, as described next.

\section{A. MPC Design}

Using the linearized HCW equations (2), the tracking error dynamics are represented by the state-space model

$$
\dot{\boldsymbol{x}}=\mathbf{A}_{c} \boldsymbol{x}+\mathbf{B}_{c} \boldsymbol{u}
$$

with

$$
\mathbf{A}_{c}=\left[\begin{array}{cccccc}
0 & 0 & 0 & 1 & 0 & 0 \\
0 & 0 & 0 & 0 & 1 & 0 \\
0 & 0 & 0 & 0 & 0 & 1 \\
0 & 0 & 0 & 0 & 0 & 2 \omega \\
0 & -\omega^{2} & 0 & 0 & 0 & 0 \\
0 & 0 & 3 \omega^{2} & -2 \omega & 0 & 0
\end{array}\right]
$$

and

$$
\mathbf{B}_{c}=\left[\begin{array}{cccccc}
0 & 0 & 0 & 1 / m & 0 & 0 \\
0 & 0 & 0 & 0 & 1 / m & 0
\end{array}\right]^{T}
$$

The system is assumed to be fully observable and hence the output is $\boldsymbol{x}(t)$. For digital implementation of the control law, the system is discretized with a sampling period $T_{s}$ using a zero-order hold, resulting in the discrete state-space model

$$
\boldsymbol{x}(k+1)=\mathbf{A} \boldsymbol{x}(k)+\mathbf{B} \boldsymbol{u}(k)
$$

where

$$
\mathbf{A}=e^{\mathbf{A}_{c} T_{s}}, \quad \mathbf{B}=\left(\int_{0}^{T_{s}} e^{\mathbf{A}_{c} \tau} \mathrm{d} \tau\right) \mathbf{B}_{c}
$$

The MPC design requires the predicted future states for a number of steps ahead, where these are generated from the state-space model $(15)$ at the current sampling instant based on the current state and the computed input sequence. Let $\boldsymbol{u}(k+j)$ denote the input to be computed $j$ sampling steps ahead from the current sampling instant $k$. The basic idea underpinning Laguerre MPC (LMPC) is to parameterize $\boldsymbol{u}(k+j)$ using a set of discrete Laguerre polynomials, as

$$
\boldsymbol{u}(k+j)=\left[\begin{array}{l}
u_{1}(k+j) \\
u_{2}(k+j)
\end{array}\right] \asymp\left[\begin{array}{cc}
\boldsymbol{l}_{1}^{T}(j) & \boldsymbol{0} \\
\boldsymbol{0} & \boldsymbol{l}_{2}^{T}(j)
\end{array}\right]\left[\begin{array}{l}
\boldsymbol{\eta}_{1} \\
\boldsymbol{\eta}_{2}
\end{array}\right]=\mathbf{L}(j) \boldsymbol{\eta}
$$

where $\boldsymbol{l}_{i}(j)$ is the Laguerre function vector and $\boldsymbol{\eta}$, which represents the new decision vector, is termed the coefficient vector.

The Laguerre function vector satisfies the difference equation

$$
\boldsymbol{l}_{i}(j+1)=\left[\begin{array}{ccccc}
a_{i} & 0 & \ldots & \ldots & 0 \\
b_{i} & a_{i} & \ddots & \vdots & 0 \\
-a_{i} b_{i} & b_{i} & \ddots & 0 & 0 \\
\vdots & \vdots & \ddots & \ddots & 0 \\
-a_{i}^{N_{i}-2} & -a_{i}^{N_{i}-3} b_{i} & \ldots & b_{i} & a_{i}
\end{array}\right] \boldsymbol{l}_{i}(j)
$$

with

$$
\boldsymbol{l}_{i}(0)=\sqrt{b_{i}}\left[\begin{array}{llllll}
1 & -a_{i} & a_{i}^{2} & -a_{i}^{3} & \ldots & (-1)^{N_{i}-1} a_{i}^{N_{i}-1}
\end{array}\right]^{T}
$$

where $b_{i}=\left(1-a_{i}^{2}\right), N_{i}$ is the number of terms in the expansion, and $a_{i} \in[0,1]$ is the scaling factor of the Laguerre network for input $u_{i}$. Both $a_{i}$ and $N_{i}$ are fixed design parameters. Setting $a_{i}=0$ in Eqs. (18) and (19), and using Eq. (17), give that 


$$
\boldsymbol{l}_{i}^{T}(j) \boldsymbol{\eta}_{i}=\left\{\begin{array}{cc}
u_{i}(k+j) & \forall j \in\left\{0 \ldots N_{i}\right\} \\
0 & \forall j>N_{i}
\end{array}\right.
$$

which corresponds to the standard MPC design with control horizon $N_{i}$. Choosing $a_{i}>0$ allows a tradeoff to be made between the time scale of the control trajectory, i.e., $\boldsymbol{l}_{i}^{T}(j) \boldsymbol{\eta}_{i}$ exponentially decays to zero instead of being identically zero for $j>N_{i}$, and the accuracy of its pointwise approximation (17). This is particularly relevant when the number of decision variables $N_{i}$ is selected to be small to keep the computation feasible and then the truncated parameterization, given by (20), cannot adequately describe the future input trajectory. Substituting Eq. (17) into Eq. (15), the state dynamics $N_{p}$ sampling instants ahead of $\bar{k}$ are given by

$$
\left\{\begin{array}{l}
\boldsymbol{x}(k+1 \mid k)=\mathbf{A} \boldsymbol{x}(k)+\mathbf{B L}(0) \boldsymbol{\eta} \\
\boldsymbol{x}(k+2 \mid k)=\mathbf{A}^{2} \boldsymbol{x}(k)+(\mathbf{A B L}(0)+\mathbf{B L}(1)) \boldsymbol{\eta} \\
\vdots \\
\boldsymbol{x}\left(k+N_{p} \mid k\right)=\mathbf{A}^{N_{p}} \boldsymbol{x}(k)+\left(\mathbf{A}^{N_{p}-1} \mathbf{B L}(0)+\cdots+\mathbf{B L}\left(N_{p}-1\right)\right) \boldsymbol{\eta}
\end{array}\right.
$$

where the prediction horizon $N_{p}$ is unrelated to the number of components of $\boldsymbol{\eta}$, which is equal to $\left(N_{1}+N_{2}\right)$.

The prediction model can be written in the compact form

$$
\boldsymbol{X}=\mathbf{F} \boldsymbol{x}(k)+\boldsymbol{\Phi} \boldsymbol{\eta}
$$

where

$$
\begin{aligned}
\boldsymbol{X} & =\left[\begin{array}{llll}
\boldsymbol{x}^{T}(k+1 \mid k) & \boldsymbol{x}^{T}(k+2 \mid k) & \ldots & \boldsymbol{x}^{T}\left(k+N_{p} \mid k\right)
\end{array}\right]^{T} \\
\mathbf{F} & =\left[\begin{array}{lllll}
(\mathbf{A})^{T} & \left(\mathbf{A}^{2}\right)^{T} & \ldots & \left(\mathbf{A}^{N_{p}}\right)^{T}
\end{array}\right]^{T} \\
\boldsymbol{\Phi} & =\left[\begin{array}{cccc}
\mathbf{B L}(0) & \mathbf{0} & \ldots & \mathbf{0} \\
\mathbf{A B L}(0) & \mathbf{B L}(1) & \cdots & \mathbf{0} \\
\vdots & \vdots & \ddots & \mathbf{0} \\
\mathbf{A}^{N_{p}-1} \mathbf{B L}(0) & \mathbf{A}^{N_{p}-2} \mathbf{B L}(1) & \cdots & \mathbf{B L}\left(N_{p}-1\right)
\end{array}\right]
\end{aligned}
$$

Moreover, the cost function Eq. (11) is discretized for $n=2$ and $N_{p}=T_{p} / T_{s}$, to give

$$
J_{d}=\boldsymbol{X}^{T} \mathbf{Q} \boldsymbol{X}+\boldsymbol{\eta}^{T} \mathbf{R} \boldsymbol{\eta}
$$

where, for the remainder of this paper, $\mathbf{W}_{f}=T_{s} \mathbf{W}$, $\mathbf{Q}=\bigoplus^{N_{p}} T_{s} \mathbf{W}^{T} \mathbf{W}$ is a $6 N_{p} \times 6 N_{p}$ matrix and $\mathbf{R}=$ $T_{s} \mathbf{M}_{u}^{T}\left(\bigoplus^{N_{p}} \mathbf{K}^{T} \mathbf{K}\right) \mathbf{M}_{u}$ is a $\left(N_{1}+N_{2}\right) \times\left(N_{1}+N_{2}\right)$ matrix, with

$$
\boldsymbol{M}_{u}=\left[\begin{array}{llll}
\mathbf{L}^{T}(0) & \mathbf{L}^{T}(1) & \ldots & \mathbf{L}^{T}\left(N_{p}-1\right)
\end{array}\right]^{T}
$$

Hence, the minimization of (24) can be equivalently rewritten as

$$
\min _{\boldsymbol{\eta}} \boldsymbol{\eta}^{T} \boldsymbol{\Omega} \boldsymbol{\eta}+2 \boldsymbol{x}^{T}(k) \boldsymbol{\Psi}^{T} \boldsymbol{\eta}
$$

where $\boldsymbol{\Omega}=\left(\boldsymbol{\Phi}^{T} \mathbf{Q} \boldsymbol{\Phi}+\mathbf{R}\right)$ and $\boldsymbol{\Psi}=\boldsymbol{\Phi} \mathbf{Q} \mathbf{F}$.

In the absence of constraints, the global minimum of problem (26) is attained (assuming the required matrix inverse exists) at

$$
\boldsymbol{\eta}^{*}(k)=-\boldsymbol{\Omega}^{-1} \boldsymbol{\Psi} \boldsymbol{x}(k)
$$

Under the receding horizon principle, only the first element of the optimal input sequence is applied to the plant and hence

$$
\boldsymbol{u}(k)=\mathbf{L}(0) \boldsymbol{\eta}^{*}(k)
$$

Input and state constraints are included in the MPC design to account for the operating range of the actuators and to ensure safe proximity operations. Unlike the unconstrained case, the constrained MPC problem does not admit an analytic solution and must be solved numerically. For a given set of samples $\mathbb{M}_{u}$, on which input constraints are enforced, Eq. (6) can be rewritten as

$$
-u_{M} \mathbf{1} \leq \mathbf{L}(j) \boldsymbol{\eta} \leq \boldsymbol{1} u_{M} \quad \forall j \in \mathbb{M}_{u} \subseteq\left\{0, \ldots, N_{p}-1\right\}
$$

To keep the computation feasible, the number of terms $N_{i}$ in Eqs. (18) and (19) must be set much lower than the number of prediction samples $N_{p}$ in Eq. (21). Therefore, in the presence of uncertain observations, the error on $\boldsymbol{x}(k)$ is propagated and the control system is required to react for potentially large deviations between the predicted state trajectories during the first few optimization samples. Consequently, the receding horizon scheme is affected by a pronounced sensitivity to high-frequency noise, which can seriously affect the tracking performance in addition to the fuel consumption and lifetime of the propulsion system. To mitigate against these effects, the input is low-pass filtered by introducing a slack variable $s_{1} \geq 0$, which represents the upper bound of the input variation over the prediction horizon, and penalizing it in the cost function.

The value of $s_{1}$ is obtained from the set of linear inequalities

$$
\begin{aligned}
& -s_{1} \boldsymbol{1} \leq \mathbf{L}(0) \boldsymbol{\eta}-\boldsymbol{u}(k-1) \leq \boldsymbol{1} s_{1} \quad j=0 \\
& -s_{1} \boldsymbol{l} \leq(\mathbf{L}(j)-\mathbf{L}(j-1)) \boldsymbol{\eta} \leq \boldsymbol{1} s_{1} \quad \forall j \in\left\{1, \ldots, N_{p}-1\right\}
\end{aligned}
$$

where $\boldsymbol{u}(k-1)$ is treated as an additional input to the optimization problem. Moreover, the nonlinear path constraints in Eq. (7) can be approximated by the following set of linear inequalities

$$
\begin{aligned}
& \mathbf{C x}(k+j \mid k) \leq 1 s_{2}+\boldsymbol{d} \quad \forall j \in \mathbb{M}_{x} \subseteq\left\{1, \ldots, N_{p}\right\} \\
& \mathbf{C}=\left[\begin{array}{cccccc}
1 & 0 & 0 & 0 & 0 & 0 \\
\tan (\theta / 2) / \sqrt{2} & 1 & 0 & 0 & 0 & 0 \\
\tan (\theta / 2) / \sqrt{2} & -1 & 0 & 0 & 0 & 0 \\
\tan (\theta / 2) / \sqrt{2} & 0 & 1 & 0 & 0 & 0 \\
\tan (\theta / 2) / \sqrt{2} & 0 & -1 & 0 & 0 & 0
\end{array}\right] \\
& \boldsymbol{d}=\left[\begin{array}{lll}
d_{1} & \boldsymbol{I}^{T} & \varepsilon
\end{array}\right]^{T}
\end{aligned}
$$

where $d_{1}, \varepsilon \geq 0$ are given tolerances and $s_{2} \geq 0$ is a slack variable that relaxes the formulation in the $\infty$ norm sense to retain the feasibility of the problem against possible conflicts between input and output constraints. Assuming $s_{2}=0, \varepsilon>0$, and $d_{1}<\sqrt{2} \varepsilon / \tan (\theta / 2)$, Eq. (31) represents a truncated pyramidal approximation of the LoS cone for the set of samples $\mathbb{M}_{x}$.

Let $\boldsymbol{\eta}_{C}=\left[\begin{array}{lll}\boldsymbol{\eta}^{T} & s_{1} & s_{2}\end{array}\right]^{T}=\left[\begin{array}{ll}\boldsymbol{\eta}^{T} & \boldsymbol{s}^{T}\end{array}\right]^{T}$ be the augmented decision vector and $\boldsymbol{x}_{C}(k)=\left[\begin{array}{ll}\boldsymbol{x}^{T}(k) & \boldsymbol{u}^{T}(k-1)\end{array}\right]^{T}$ be the augmented input in the constrained optimization problem and assume, for notational simplicity, that Eqs. (29) and (31) are defined for all samples. The constraints (29), (30), and (31) can hence be written in the compact form

$$
\begin{aligned}
& {\left[\begin{array}{ccc}
\mathbf{M}_{u} & \boldsymbol{0} & \boldsymbol{0} \\
-\mathbf{M}_{u} & \boldsymbol{0} & \boldsymbol{0} \\
\mathbf{M}_{\Delta} & -\boldsymbol{1} & \boldsymbol{0} \\
-\mathbf{M}_{\Delta} & -\boldsymbol{1} & \boldsymbol{0} \\
\mathbf{C}_{N} \boldsymbol{\Phi} & \mathbf{0} & -\boldsymbol{1}
\end{array}\right]\left[\begin{array}{c}
\boldsymbol{\eta} \\
s_{1} \\
s_{2}
\end{array}\right]} \\
& \leq\left[\begin{array}{c}
\boldsymbol{1} u_{M} \\
\boldsymbol{1} u_{M} \\
\boldsymbol{0} \\
\boldsymbol{0} \\
\boldsymbol{d}_{N}
\end{array}\right]-\left[\begin{array}{rr}
\mathbf{0} & \mathbf{0} \\
\mathbf{0} & \mathbf{0} \\
\mathbf{0} & -\mathbf{U}_{\Delta} \\
\mathbf{0} & \mathbf{U}_{\Delta} \\
\mathbf{C}_{N} \mathbf{F} & \mathbf{0}
\end{array}\right]\left[\begin{array}{c}
\boldsymbol{x}(k) \\
\boldsymbol{u}(k-1)
\end{array}\right]
\end{aligned}
$$

where $\mathbf{F}$ and $\boldsymbol{\Phi}$ are given by Eq. (23) $, \mathbf{C}_{N}=\bigoplus^{N_{p}} \mathbf{C}, \boldsymbol{d}_{N}=$ $\left[\begin{array}{lll}\boldsymbol{d}^{T} & \ldots & \boldsymbol{d}^{T}\end{array}\right]^{T}$ and 


$$
\begin{aligned}
\mathbf{M}_{\Delta} & =\left[\begin{array}{llll}
\mathbf{L}^{T}(0) & \mathbf{L}^{T}(1)-\mathbf{L}^{T}(0) & \ldots & \mathbf{L}^{T}\left(N_{p}-1\right)-\mathbf{L}^{T}\left(N_{p}-2\right)
\end{array}\right]^{T} \\
\mathbf{U}_{\Delta} & =\left[\begin{array}{llll}
\mathbf{I} & \mathbf{0} & \ldots & \mathbf{0}
\end{array}\right]^{T}
\end{aligned}
$$

The constrained LMPC problem of the form (26) to be solved is

$$
\begin{array}{ll}
\min _{\eta_{C}} & \boldsymbol{\eta}_{C}^{T}\left[\begin{array}{cc}
\boldsymbol{\Omega} & \mathbf{0} \\
\mathbf{0} & \mathbf{R}_{s}
\end{array}\right] \boldsymbol{\eta}_{C}+2 \boldsymbol{x}_{C}^{T}(k)\left[\begin{array}{cc}
\boldsymbol{\Psi}^{T} & \mathbf{0} \\
\mathbf{0} & \mathbf{0}
\end{array}\right] \boldsymbol{\eta}_{C} \\
\text { s.t. } & (32)
\end{array}
$$

where $\mathbf{R}_{s}$ is a $2 \times 2$ positive definite diagonal matrix that heavily penalizes the slack vector $\boldsymbol{s}$. Problem (34) is solved at each sampling instant $k$, yielding $\boldsymbol{\eta}_{C}^{*}(k)$, and the first element of the control sequence is applied to the plant via Eq. (28).

\section{B. Explicit LMPC}

Even if the optimization problem (34) can be solved efficiently using existing quadratic programming $(\mathrm{QP})$ algorithms, the required computations may not be feasible for spacecraft with low processing power. Moreover, the execution time of QP solvers is in general not guaranteed, whereas the reliability of the control system is a primary concern for space applications. In this respect, one possibility is to use explicit MPC.

Prior to proceeding, it is useful to rewrite the constrained LMPC problem in terms of the simplified notation

$$
\begin{array}{cl}
\min _{\boldsymbol{\eta}_{C}} & \boldsymbol{\eta}_{C}^{T} \mathbf{H} \boldsymbol{\eta}_{C}+2 \boldsymbol{x}_{C}^{T}(k) \mathbf{G} \boldsymbol{\eta}_{C} \\
\text { s.t. } & \mathbf{M} \boldsymbol{\eta}_{C} \leq \mathbf{D}+\mathbf{E} \boldsymbol{x}_{C}(k)
\end{array}
$$

where the matrices $\mathbf{H}, \mathbf{G}, \mathbf{M}, \mathbf{D}$, and $\mathbf{E}$ are obtained from Eqs. (32) and (34). On defining the new variable

$$
z \triangleq \boldsymbol{\eta}_{C}+\mathbf{H}^{-1} \mathbf{G}^{T} \boldsymbol{x}_{C}(k)
$$

Eq. (35) can be transformed by completing squares into the equivalent multiparametric quadratic program

$$
\begin{array}{ll}
\min _{z} & z \mathbf{H} z \\
\text { s.t. } & \mathbf{M} z \leq \mathbf{D}+\left(\mathbf{E}+\mathbf{M H}^{-1} \mathbf{G}^{T}\right) \boldsymbol{x}_{C}(k)
\end{array}
$$

where $\boldsymbol{x}(k)$, which appears only in the right-hand side of the equation, is treated as a parameter vector.

Problem (37) can be solved explicitly for all the parameters $\boldsymbol{x}_{C}(k)$ inside a given polyhedral set $\overline{\mathbb{X}}_{C}$, as described in, for example, [22]. For the proposed MPC design, it is beneficial to consider a region of additional size $\boldsymbol{d}_{s} \geq \boldsymbol{0}$ with respect to the set defined by Eq. (31), together with the maximum excursion of the control. Because the resulting set is not closed, auxiliary bounds are specified for the along-track position and the velocity tracking errors using

$$
\mathbf{C}_{a} \boldsymbol{x}(k)=\left[\begin{array}{cccccc}
-1 & 0 & 0 & 0 & 0 & 0 \\
k_{1} & 0 & 0 & 1 & 0 & 0 \\
k_{1} & 0 & 0 & -1 & 0 & 0 \\
k_{2} & 0 & 0 & 0 & 1 & 0 \\
k_{2} & 0 & 0 & 0 & -1 & 0 \\
k_{3} & 0 & 0 & 0 & 0 & 1 \\
k_{3} & 0 & 0 & 0 & 0 & -1
\end{array}\right] \boldsymbol{x}(k) \leq \boldsymbol{d}_{a}=\left[\begin{array}{c}
x_{M} \\
1 \varepsilon_{a}
\end{array}\right]
$$

where $x_{M}$ is the maximum feasible along-track separation between the two spacecraft, $\varepsilon_{a} \geq 0$ is a specified tolerance, and $k_{1}, k_{2}$, and $k_{3}$ are positive slopes. The linear dependence of the velocities on $x_{1}$ is justified by collision avoidance requirements and by the linear dependence of the LoS constraints on $x_{1}$, and the final form of the parameter space is

$$
\overline{\mathbb{X}}_{C}=\left\{\boldsymbol{x}_{C}(k):\left[\begin{array}{cc}
\mathbf{C} & \mathbf{0} \\
\mathbf{0} & \mathbf{I} \\
\mathbf{0} & -\mathbf{I} \\
\mathbf{C}_{a} & \mathbf{0}
\end{array}\right] \boldsymbol{x}_{C}(k) \leq\left[\begin{array}{c}
\boldsymbol{d}+\boldsymbol{d}_{s} \\
\boldsymbol{1} u_{M} \\
\mathbf{1} u_{M} \\
\boldsymbol{d}_{a}
\end{array}\right]\right\}
$$

The solution $z^{*}\left(x_{C}(k)\right)$ of Eq. (37) is a piecewise affine linear function defined over a polyhedral partition of $\overline{\mathbb{X}}_{C}$, which can be stored in look-up table form. At each sampling instant, the thrust command is

$$
\boldsymbol{u}(k)=\left[\begin{array}{ll}
\mathbf{L}(0) & \mathbf{0}
\end{array}\right]\left(z^{*}\left(\boldsymbol{x}_{C}(k)\right)-\mathbf{H}^{-1} \mathbf{G}^{T} \boldsymbol{x}_{C}(k)\right)
$$

where the online computational load is limited to a piecewise affine function evaluation. This requirement consists of locating the statespace region and hence the look-up table entry that contains the precomputed control law for a given $\boldsymbol{x}_{C}(k)$, through the solution of a set-membership problem.

\section{Reference Mission}

A possible scenario for the application of the LMPC design developed in this paper is a low-Earth-orbit formation flying mission performed by two cubesat size spacecraft, where the relative dynamics are controlled by means of a miniaturized electric propulsion system. At the beginning of the operative phase, the spacecraft are flying in a near circular polar orbit, at an altitude of approximately $450 \mathrm{~km}$. The leader and follower spacecraft have identical physical parameters: the total mass of each is $3 \mathrm{~kg}$, the bus size is $30 \times 10 \times 10 \mathrm{~cm}^{3}$, and the cross-sectional area is $10 \times 10 \mathrm{~cm}^{2}$. The electric propulsion system considered in this work is a set of pulsed plasma thrusters (PPT) specifically designed for application to cubesats, as described in [35]. Table 1 gives the characteristics of the PPT model.

The relative position observation model is based on the specifications of differential GPS and optical sensors [ $\underline{36}, \underline{37}]$, where the field of view of the optical sensor is assumed to be $\bar{\theta}=30 \mathrm{deg}$. The standard deviation of the observation uncertainty is given, as a function of the along-track separation between the two spacecraft, in Fig. 2. Relative velocity is estimated from relative position observations using a symmetric finite impulse response filter of order 8. Based on these observations, the follower spacecraft is required to dock with the leader whilst satisfying LoS and input constraints, using two pairs of opposite PPTs aligned with the along-track and cross-track directions.

A high-accuracy nonlinear simulation model has been developed for the considered scenario. The state vector of the model includes the position and velocity vectors for the leader $\left(\boldsymbol{r}_{L}, \boldsymbol{v}_{L}\right)$ and the follower $\left(\boldsymbol{r}_{F}, \boldsymbol{v}_{F}\right)$. The equations that describe the evolution of the state vector in the Earth-centered inertial (ECI) frame are

$$
\begin{gathered}
\dot{\boldsymbol{r}}_{L}=\boldsymbol{v}_{L} \\
\dot{\boldsymbol{v}}_{L}=-\frac{\mu}{r_{L}^{3}} \boldsymbol{r}_{L}+\boldsymbol{a}_{L}
\end{gathered}
$$

Table 1 PPT specifications

\begin{tabular}{lc}
\hline \hline Parameter & Value \\
\hline Mass & $180 \mathrm{~g}($ wet mass $)+90 \mathrm{~g}$ (electronics) \\
Dimensions & $90.17 \times 95.89 \times 31 \mathrm{~mm}$ \\
Impulse bit & $40 \mu \mathrm{Ns}$ \\
Pulse frequency & $\leq 1 \mathrm{~Hz}$ \\
Total impulse & $42 \mathrm{Ns}$ \\
Specific impulse & $608 \mathrm{~s}$ \\
Power & $0.3-4 \mathrm{~W}$ \\
Misalignment & 1 deg per axis \\
Noise, std. dev. & $5 \%$ of nominal impulse \\
\hline \hline
\end{tabular}




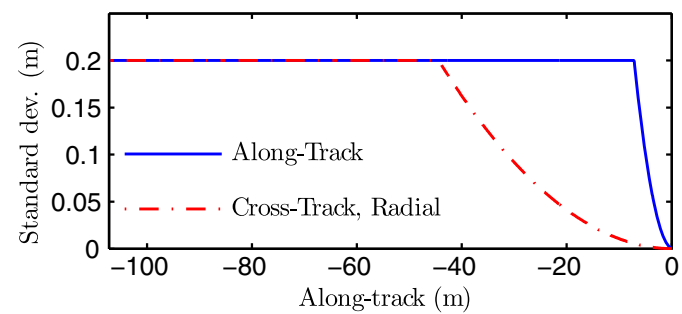

Fig. 2 Standard deviation of observation uncertainty.

$$
\begin{gathered}
\dot{\boldsymbol{r}}_{F}=\boldsymbol{v}_{F} \\
\dot{\boldsymbol{v}}_{F}=-\frac{\mu}{r_{F}^{3}} \boldsymbol{r}_{F}+\boldsymbol{a}_{F}, \quad \boldsymbol{v}_{F}^{+}=\boldsymbol{v}_{F}^{-}+\Delta \boldsymbol{v}_{F}
\end{gathered}
$$

where $\mu$ is the gravitational parameter of the Earth and Eq. (44) accounts for the impulsive change $\Delta \boldsymbol{v}_{F}$ of the follower velocity due to PPT operation. The calculation of the disturbance accelerations $\boldsymbol{a}_{L}$ and $\boldsymbol{a}_{F}$ is based on the main orbital perturbations acting on spacecraft at low altitudes. A spherical harmonic expansion up to degree and order 9 is used for the Earth's gravity field [38]. The drag force is calculated using a drag coefficient of 2.5 and the Jacchia- 71 model to approximate the atmospheric density [39]. A cannonball model is employed for the calculation of the solar radiation force, taking into account eclipse conditions. Disturbance accelerations due to the point-mass lunar and solar gravity fields are also considered, where the position of the Sun and Moon is obtained through precise ephemerides. True relative position and velocity are expressed in the LVLH frame as

$$
\begin{gathered}
\delta \boldsymbol{r}=\mathbf{R}_{L}^{I}\left(\boldsymbol{r}_{F}-\boldsymbol{r}_{L}\right) \\
\delta \dot{\boldsymbol{r}}=\mathbf{R}_{L}^{I}\left(\boldsymbol{v}_{F}-\boldsymbol{v}_{L}\right)-[\boldsymbol{\omega} \times] \mathbf{R}_{L}^{I}\left(\boldsymbol{r}_{F}-\boldsymbol{r}_{L}\right)
\end{gathered}
$$

where $\mathbf{R}_{L}^{I}$ is the matrix that represents the coordinate transformation between the inertial and LVLH frames and $[\boldsymbol{\omega} \times]$ is the skewsymmetric matrix of $\omega=\left[\begin{array}{lll}0 & \omega & 0\end{array}\right]^{T}$. The LVLH rate is given by

$$
\omega=-\sqrt{\frac{\mu}{\left\|r_{L}\right\|_{2}^{3}}}
$$

An integral pulse frequency modulator is used to translate the piecewise constant command signal from Eq. (40) into discrete pulses, as required for PPT operation. The modulator delivers a pulse $p$ whenever the integral of the commanded thrust $U(t)$ is greater or equal to the impulse bit $U_{M}$ of the thrusters. For each component of the input

$$
U_{i}\left(t_{k}\right)=U_{i}\left(t_{k-1}\right)+\frac{u_{i}\left(t_{k-1}\right)+u_{i}\left(t_{k}\right)}{2} \Delta t
$$

where $\Delta t=t_{k}-t_{k-1}$ is the modulator step size, the modulator output is calculated as

$$
p_{i}\left(t_{k}\right)=\left\{\begin{array}{cl}
U_{M} \operatorname{sgn}\left(U_{i}\left(t_{k}\right)\right) & \text { if }\left|U_{i}\left(t_{k}\right)\right| \geq U_{M} \\
0 & \text { if }\left|U_{i}\left(t_{k}\right)\right|<U_{M}
\end{array}\right.
$$

and the update

$$
U_{i}\left(t_{k}\right)=U_{i}\left(t_{k}\right)-p_{i}\left(t_{k}\right)
$$

is used in the calculation of $U_{i}\left(t_{k+1}\right)$. Under the assumption that the attitude of the spacecraft is controlled to match the orientation of the LVLH frame, the impulsive velocity change, expressed in the inertial frame, is given by

$$
\Delta \boldsymbol{v}_{F}=\mathbf{R}_{I}^{L} \delta \mathbf{R} \frac{\boldsymbol{p}+\boldsymbol{w}}{m}
$$

where $\mathbf{R}_{I}^{L}=\left(\mathbf{R}_{L}^{I}\right)^{T}, \boldsymbol{p}=\left[\begin{array}{lll}p_{1} & p_{2} & 0\end{array}\right]^{T}, \delta \mathbf{R}$ denotes the thruster alignment error, and $\boldsymbol{w}=\left[\begin{array}{lll}w_{1} & w_{2} & 0\end{array}\right]^{T}$ represents the thruster noise.

\section{Simulations}

In this section, the results from a simulation case study of the performance of the proposed design are given, including a comparison with standard MPC and LQR techniques and a feasibility assessment for the reference mission. The control law is tuned to tradeoff between the maneuver time and the fuel consumption. Even if these quantities do not explicitly appear in the approximation (24) of the original cost function (8), the system is null controllable with vanishing input energy, from which it follows that, for a sufficiently long prediction horizon and a relatively small state penalty compared to the input penalty, the minimum energy solution approaches the minimum fuel solution $[27,33]$. Moreover, a small penalty on the state allows for a reduction of the number of input constraints in the MPC optimization problem, simplifying its solution.

The elimination of radial thrust, which is an underlying assumption of this work, has proven to be effective in improving the fuel efficiency of control laws based on a quadratic performance index [40]. Because the cross-track motion is a simple undamped oscillatory motion that is decoupled from the rest of the system, pure derivative control can be applied on this axis to provide adequate damping [41]. Equivalently, the cross-track position weighting can be set to zero in Eq. (24). Finally, the control gains can be appropriately scaled by the mean motion according to [42]. These guidelines are useful to identify a suitable initial set of tuning parameters, whilst their fine tuning relies on a trial-and-error procedure based on numerical simulations. Table $\underline{2}$ gives the tuning parameters used in the simulations.

An explicit control law can be computed offline by solving the associated multiparametric quadratic program for the parameter space resulting from use of the parameters given in Table $\underline{3}$. The solution is a polyhedral partition of the parameter space defined by 946 regions in 8 dimensions (six states for the relative motion plus two previous inputs). The real-time computation of the control law onboard the spacecraft is conveniently reduced to a set-membership evaluation and the step size of the integral pulse frequency modulator is taken as $\Delta t=1 \mathrm{~s}$, according to the thruster specifications in Table 1

Table 2 LMPC tuning parameters

\begin{tabular}{lc}
\hline \hline Parameter & Value \\
\hline Position weight & $\mathbf{W}=\operatorname{diag}(0.8,0,1,1 / \omega, 3 / \omega, 1 / \omega)$ \\
Input weight & $\mathbf{K}=\mathbf{I} / \omega^{2}$ \\
Slack weight & $\mathbf{R}_{s}=\operatorname{diag}\left(10^{14}, 10^{5}\right)$ \\
Sampling time & $T_{s}=10 \mathrm{~s}$ \\
Prediction horizon & $N_{p}=1000$ \\
Laguerre terms & $N_{1}=N_{2}=4$ \\
Scaling factor & $a_{1}=a_{2}=0.67$ \\
Input constraint & $u_{M}=40 \mu \mathrm{N}_{1}, \mathbb{M}_{u}=\{0\}$ \\
Output constraint & $d_{1}=\varepsilon=2 \mathrm{~cm}, \mathbb{M}_{x}=\{1,150\}$ \\
\hline \hline
\end{tabular}

Table 3 Parameters of explicit LMPC

\begin{tabular}{lc}
\hline \hline Parameter & Value \\
\hline Max. feasible separation & $x_{M}=350 \mathrm{~m}$ \\
Additional LoS region & $\boldsymbol{d}_{s}=[0.1,10,10,10,10]^{T} \mathrm{~m}$ \\
Velocity slopes & $k_{1}=0.002, k_{2}=k_{3}=0.001$ \\
Velocity tolerance & $\varepsilon_{a}=0.5 \mathrm{~mm} / \mathrm{s}$ \\
\hline \hline
\end{tabular}




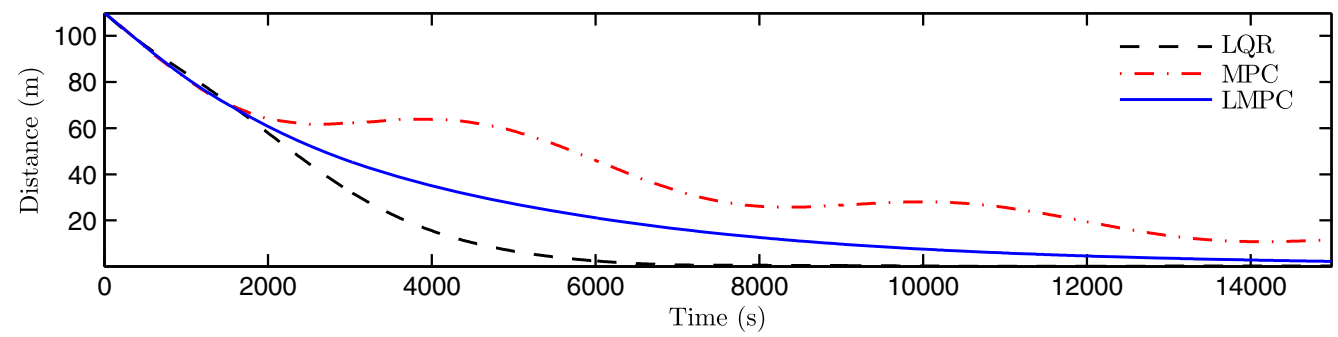

Fig. 3 Tracking performance.
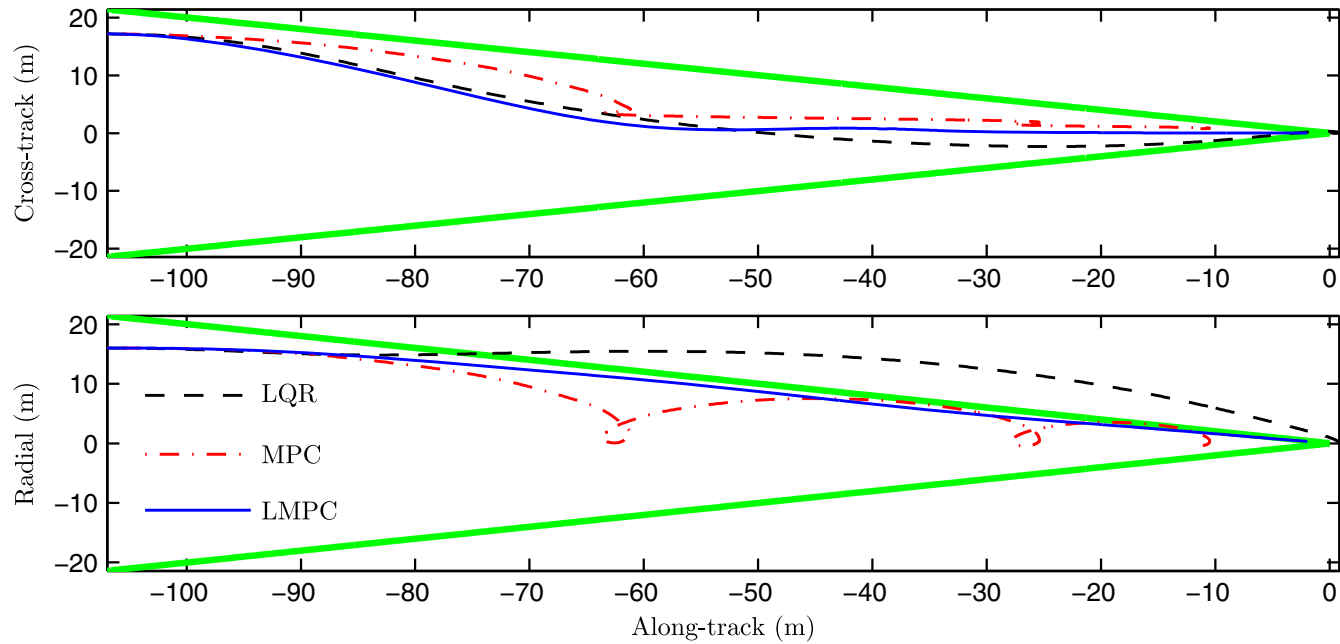

Fig. 4 LoS constraints (shown in bold) and relative trajectory.

\section{A. Control Law Comparison}

In this section, the LMPC control law is compared to an LQR and a standard MPC law with equal input and output gains, by application to the linearized HCW model (2). In this study, the standard MPC formulation is recovered from the LMPC scheme by setting the scaling factors $a_{1}, a_{2}$ of the Laguerre function network to zero.

Figure 3 gives the results for the three controllers in terms of the magnitude of the tracking error for a sample rendezvous and docking maneuver. As expected, the fastest convergence is achieved by the LQR controller, which does not enforce input and output constraints, whereas the LMPC scheme shows a much better transient response than the standard MPC scheme. In particular, the oscillatory behavior of the closed-loop system due to myopic parameterization of the input sequence is avoided. The superior performance of the LMPC scheme is supported by the fact that the explicit solution to the standard MPC problem requires a larger number (1015) of regions. The horizontal plane and the in-plane motions are shown in Fig. 4, together with the sections of the pyramid that approximate the LoS cone. Evidently, the LQR controller is unable to keep the radial tracking error within the LoS constraints.

Figure 5 gives the thrust profiles calculated by each control law. During the initial phase of the maneuver, the along-track LQR
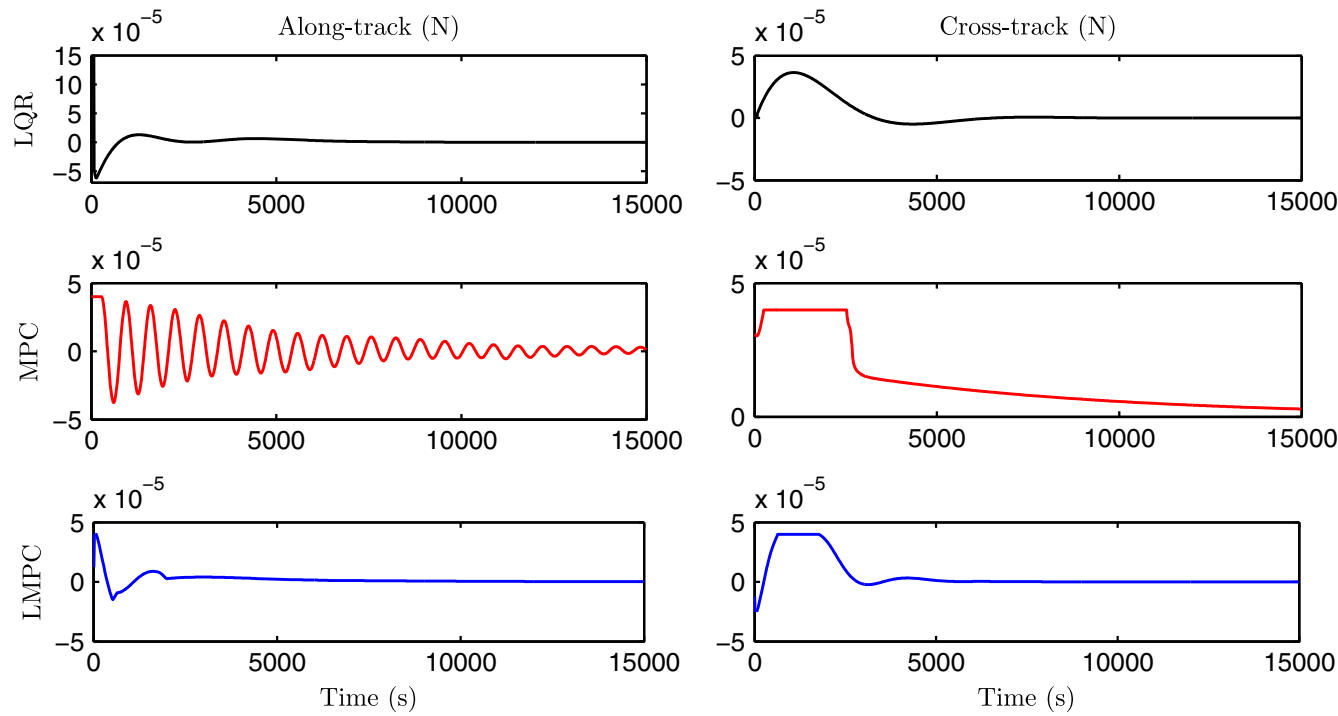

Fig. 5 Thrust profile. 
Table 4 Total impulse sensitivity to observation uncertainty

\begin{tabular}{lccc}
\hline \hline Type & LQR & MPC & LMPC \\
\hline Without noise & $0.146 \mathrm{Ns}$ & $0.330 \mathrm{Ns}$ & $0.121 \mathrm{Ns}$ \\
With noise & $0.2420 \mathrm{Ns}$ & $0.5320 \mathrm{Ns}$ & $0.1591 \mathrm{Ns}$ \\
Difference & $+65 \%$ & $+62 \%$ & $+31 \%$ \\
\hline \hline
\end{tabular}

command exceeds the maximum thrust that can be delivered by the propulsion system. Because the magnitude of the input is hardconstrained in the model predictive framework, both the MPC and the LMPC commands do not exceed the maximum operating range of the actuators. The thrust profiles calculated from uncertain observations (see Fig. 2) are given in Fig. 6. Comparing these results with the ideal case in Fig. 5, it is evident that the use of Laguerre functions, in combination with an appropriate weight on the input variation, provides the lowest sensitivity to observation uncertainty. This is confirmed by Table 4, which reports the total impulse and hence the fuel consumption sensitivity to the uncertainty. The performance degradation is approximately $60 \%$ for both the LQR and the standard MPC schemes, but $31 \%$ for the LMPC design.

\section{B. Docking Maneuver Simulation}

A number of docking maneuvers have been simulated using the nonlinear formation flying model (41-44), which includes the PPT model, in combination with the observation model in Fig. 2 and the LMPC control law. The set of initial conditions for which the relative motion lies near the edge of the LoS region has been identified as the worst-case simulation scenario. Two representative simulation cases are reported, with equal along-track initial separation and opposite initial conditions for the cross-track and radial components of the relative position vector. The initial conditions of case 1 are the same as those used for the comparison of control laws.

Figures $\underline{7}$ and $\underline{8}$ show that the LMPC control law is able to drive the follower spacecraft to the docking position while satisfying the LoS constraints in both cases. The magnitude of the relative position vector at the end of the simulation is equal to $9 \mathrm{~mm}$ for case 1 and $4 \mathrm{~cm}$ for case 2 . The good agreement of the case 1 results with those for the linear simulations (Figs. 3 and 4) suggests an appreciable robustness of the control system with respect to perturbations.

The PPT pulse profile is given in Fig. 9, together with the LMPC command, for case 1 (similar results were obtained for case 2). These results show almost no impulses are commanded in the negative along-track direction during the final phase of the approach, which indicates that plume impingement is avoided according to Eq. (9).

As a final comparison in this paper, the results obtained are compared with the open-loop solution of Eq. (10). To enable this comparison, the boundary value problem (10) is solved using the commercial package DIDO, which implements pseudospectral (PS) methods [14]. A value of $\alpha=1$ and $\beta=0$ is set in the cost function $(8)$, with the terminal time $t_{f}$ and terminal condition $\boldsymbol{x}\left(t_{f}\right)$ fixed as in the previous simulations. A good approximation of the optimal trajectory is obtained using 30 nodes for the PS solution.

The open-loop (PS) and feedback (LMPC) solutions are compared in Table 5, in terms of the total impulse required for the docking maneuver and the evaluation time of the control law on a $2 \mathrm{GHz}$ single-core CPU. Note that the total impulse commanded by the
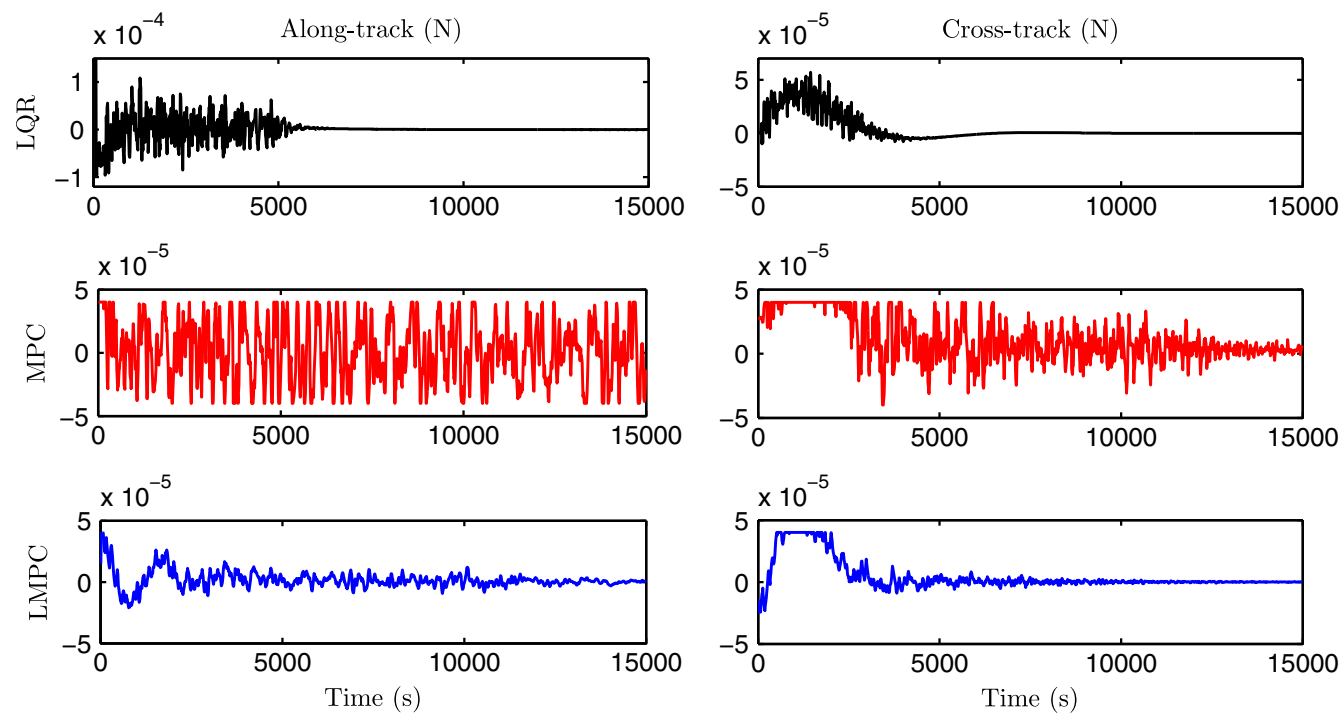

Fig. 6 Thrust profile from uncertain observations.

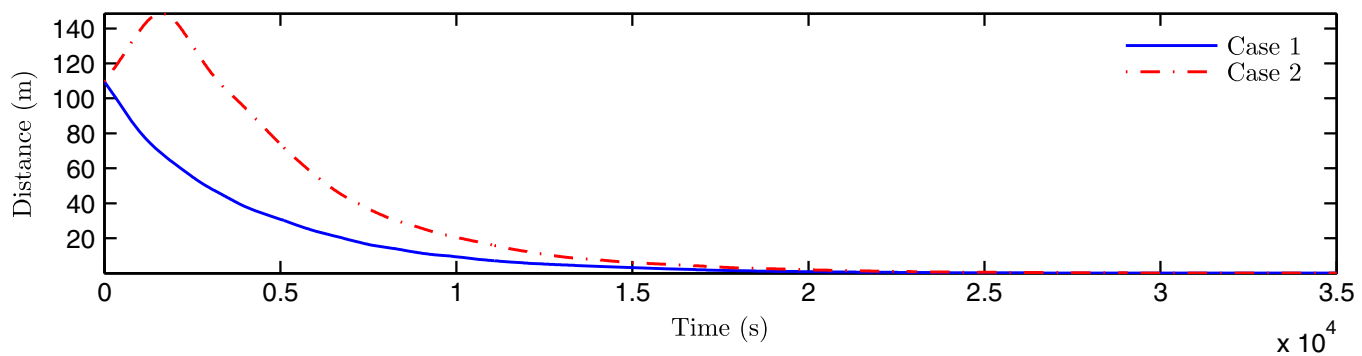

Fig. 7 LMPC tracking performance. 

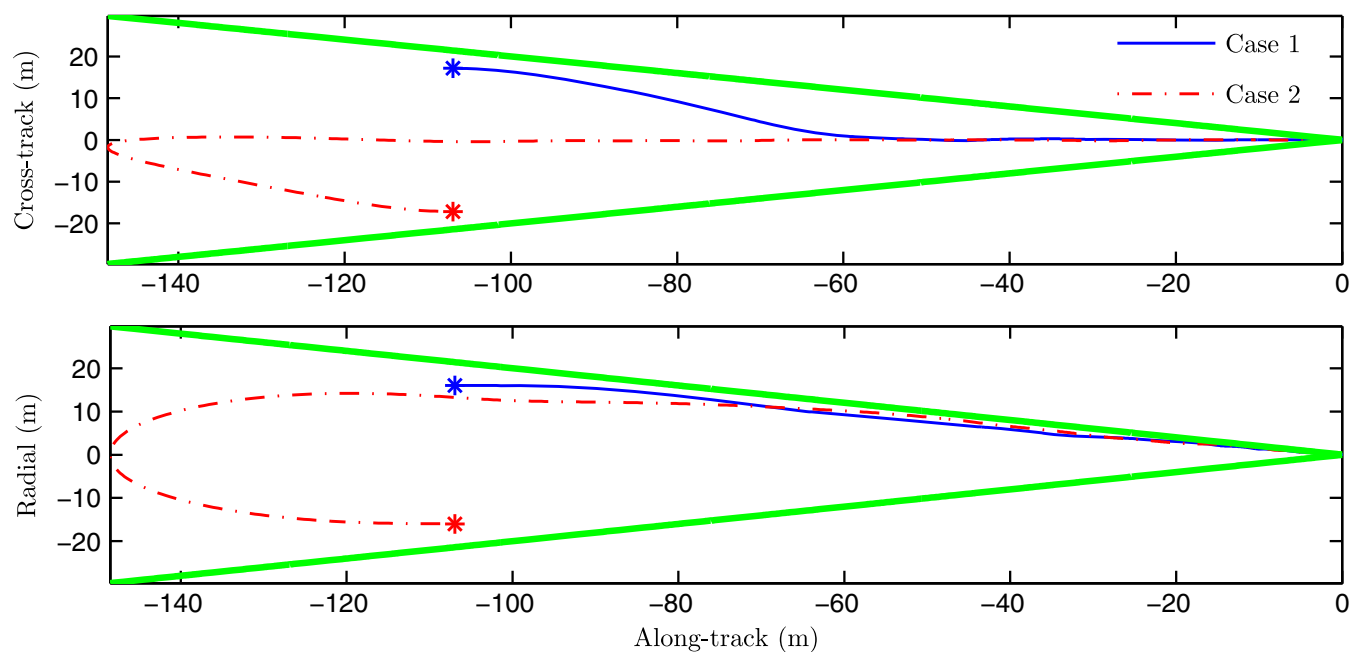

Fig. 8 LoS constraints (shown in bold) and LMPC trajectory.
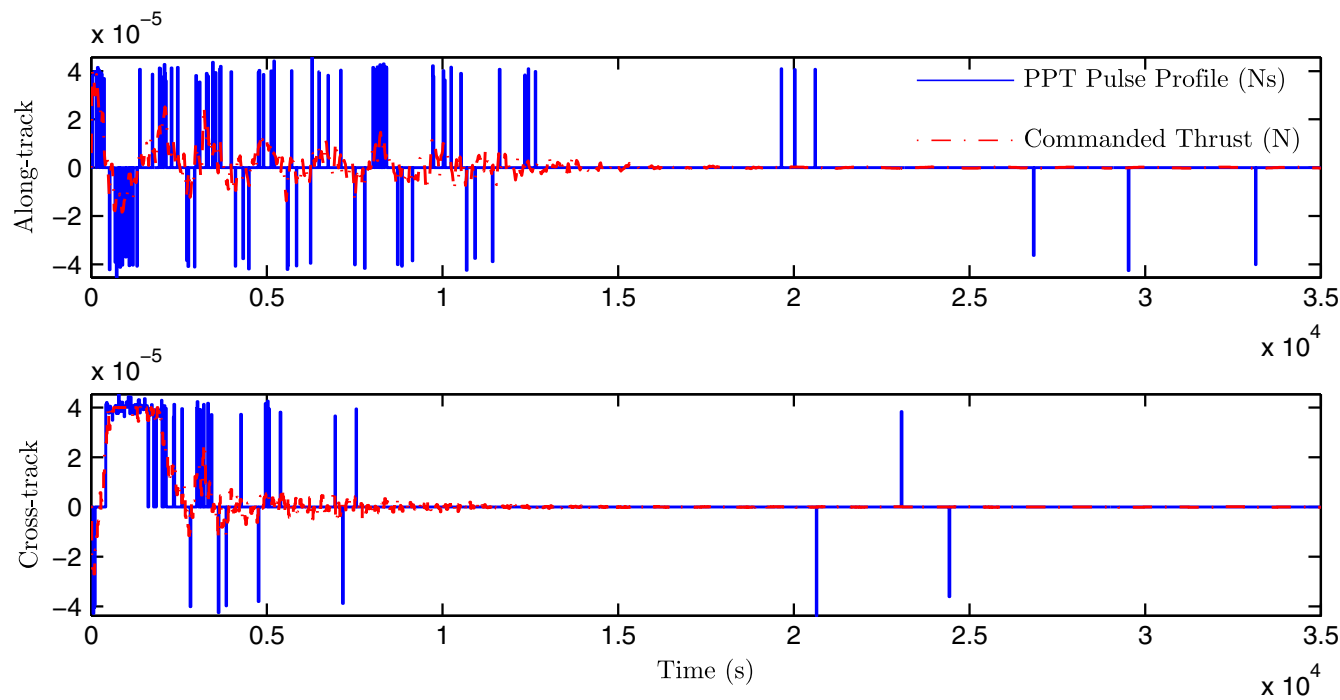

Fig. 9 PPT pulse profile and commanded thrust for case 1.

feedback system can be more than twice the one calculated by the open-loop strategy, which represents the (approximated) lower bound for the considered scenario. According to Table 4, a significant part of this mismatch is due to uncertain observations, whereas the rest arises from the nonlinearities and perturbations included in the closed-loop simulation model, and the approximations made in the design of the LMPC scheme to retain a sufficiently low computational complexity. In fact, the explicit LMPC solution is evaluated approximately 400 times faster than the PS solution. Given the relatively highly specific impulse of PPTs compared to traditional microthrusters, the new design allows a tradeoff to be made between the performance and the computational burden of the control law.

Table 5 Open-loop (PS) and feedback solution (LMPC)

\begin{tabular}{lccc}
\hline \hline Type & Case 1 impulse & Case 2 impulse & Running time \\
\hline PS & $0.09 \mathrm{Ns}$ & $0.11 \mathrm{Ns}$ & $\sim 20 \mathrm{~s}$ \\
LMPC & $0.16 \mathrm{Ns}$ & $0.25 \mathrm{Ns}$ & $\sim 0.05 \mathrm{~s}$ \\
Difference & $+77 \%$ & $+127 \%$ & $1 / 400$ \\
\hline \hline
\end{tabular}

\section{Conclusions}

This paper has demonstrated that the use of Laguerre functions in combination with multiparametric programming techniques can be effective in improving the computational efficiency of MPC when applied to the problem of low-thrust spacecraft rendezvous and proximity operations. The new design is general enough to systematically handle path constraints, as well as thrust magnitude and rate constraints. By use of the Laguerre parameterization of the input trajectories, a long planning horizon can be addressed by solving the control problem explicitly, thus circumventing the need for a dedicated solver onboard the spacecraft. Simulation results show that the achievable performance, in terms of control accuracy and propellant usage, is suitable for autonomous rendezvous and docking between small three-axis stabilized spacecraft using electric propulsion. However, the applicability of the new design to more complex scenarios, such as circumnavigation and docking with a tumbling target, still needs a deeper investigation.

\section{Acknowledgments}

The authors would like to thank A. Garulli and A. Giannitrapani for the fruitful discussion. 


\section{References}

[1] Gill, E., Montenbruck, O., and D'Amico, S., "Autonomous Formation Flying for the PRISMA Mission," Journal of Spacecraft and Rockets, Vol. 44, No. 3, 2007, pp. 671-681. doi: $10.2514 / 1.23015$

[2] Sjoberg, F., Karlsson, A., and Jakobsson, B., "PROBA-3: A Formation Flying Demonstration Mission on the Verge to be Realised," Proceedings of the 3rd International Symposium on Formation Flying, Missions and Technologies, ESA Communication Production Office, ESTEC, Noordwijk, The Netherlands, 2008; also ESA SP-654.

[3] Fridlund, C., "DARWIN-The Infrared Space Interferometry Mission," ESA Bulletin, Vol. 103, No. 3, 2000, pp. 20-25.

[4] Mattingly, R., Hayati, S., and Udomkesmalee, G., "Technology Development Plans for the Mars Sample Return Mission," Aerospace Conference, IEEE, Piscataway, NJ, 2005, pp. 982-995. doi:10.1109/AERO.2005.1559389

[5] Personne, G., Lopez-Y-Diaz, A., and Delpy, P., "ATV GNC Synthesis: Overall Design, Operations and Main Performances," Proceedings of the 6th International ESA Conference on Guidance, Navigation and Control Systems, ESA Publ. Division, ESTEC, Noordwijk, The Netherlands, 2006; also ESA SP-606.

[6] Kaiser, C., Sjöberg, F., Delcura, J. M., and Eilertsen, B., "SMART-OLEVAn Orbital Life Extension Vehicle for Servicing Commercial Spacecrafts in GEO," Acta Astronautica, Vol. 63, No. 1, 2008, pp. 400-410. doi:10.1016/j.actaastro.2007.12.053

[7] Guelman, M., and Aleshin, M., "Optimal Bounded Low-Thrust Rendezvous with Fixed Terminal-Approach Direction," Journal of Guidance, Control, and Dynamics, Vol. 24, No. 2, 2001, pp. 378-385. doi: $10.2514 / 2.4722$

[8] Bevilacqua, R., Hall, J. S., and Romano, M., "Multiple Spacecraft Rendezvous Maneuvers by Differential Drag and Low Thrust Engines," Celestial Mechanics and Dynamical Astronomy, Vol. 106, No. 1, 2010, pp. $69-88$. doi:10.1007/s10569-009-9240-3

[9] Garulli, A., Giannitrapani, A., Leomanni, M., and Scortecci, F., "Autonomous Low-Earth-Orbit Station-Keeping with Electric Propulsion," Journal of Guidance, Control, and Dynamics, Vol. 34, No. 6, 2011, pp. 1683-1693. doi:10.2514/1.52985

[10] Mazal, L., Mingotti, G., and Gurfil, P., "Optimal On-Off Cooperative Maneuvers for Long-Term Satellite Cluster Flight," Journal of Guidance, Control, and Dynamics, Vol. 37, No. 2, 2014, pp. 391-402. doi:10.2514/1.61431

[11] Breger, L. S., and How, J. P., "Safe Trajectories for Autonomous Rendezvous of Spacecraft," Journal of Guidance, Control, and Dynamics, Vol. 31, No. 5, 2008, pp. 1478-1489. doi: $10.2514 / 1.29590$

[12] Wertz, J. R., and Bell, R., "Autonomous Rendezvous and Docking Technologies: Status and Prospects," Proceedings of the Society of Photo-Optical Instrumentation Engineers: Space Systems Technology and Operations, Vol. 5088, edited by Tchoryk, P., Jr., and Shoemaker, J., Soc. of Photo-Optical Instrumentation Engineers (Int. Soc. for Optical Engineering), Bellingham, WA, 2003, pp. 20-30.

[13] Betts, J. T., "Survey of Numerical Methods for Trajectory Optimization," Journal of Guidance, Control, and Dynamics, Vol. 21, No. 2, 1998, pp. 193-207. doi: $10.2514 / 2.4231$

[14] Ross, I. M., and Fahroo, F., "Pseudospectral Knotting Methods for Solving Nonsmooth Optimal Control Problems," Journal of Guidance, Control, and Dynamics, Vol. 27, No. 3, 2004, pp. 397-405. doi: $10.2514 / 1.3426$

[15] Epenoy, R., "Fuel Optimization for Continuous-Thrust Orbital Rendezvous with Collision Avoidance Constraint," Journal of Guidance, Control, and Dynamics, Vol. 34, No. 2, 2011, pp. 493-503. doi:10.2514/1.50996

[16] Richards, A., and How, J. P., "Model Predictive Control of Vehicle Maneuvers with Guaranteed Completion Time and Robust Feasibility," Proceedings of the American Control Conference, Vol. 5, IEEE, Piscataway, NJ, 2003, pp. 4034-4040. doi:10.1109/ACC.2003.1240467

[17] Breger, L., How, J., and Richards, A., "Model Predictive Control of Spacecraft Formations with Sensing Noise," Proceedings of the American Control Conference, IEEE, Piscataway, NJ, 2005, pp. 23852390.

doi:10.1109/ACC.2005.1470323

[18] Di Cairano, S., Park, H., and Kolmanovsky, I., "Model Predictive Control Approach for Guidance of Spacecraft Rendezvous and Proximity Maneuvering," International Journal of Robust and
Nonlinear Control, Vol. 22, No. 12, 2012, pp. 1398-1427. doi: $10.1002 /$ rnc. 2827

[19] Hartley, E. N., Trodden, P. A., Richards, A. G., and Maciejowski, J. M., "Model Predictive Control System Design and Implementation for Spacecraft Rendezvous," Control Engineering Practice, Vol. 20, No. 7 , 2012, pp. 695-713. doi:10.1016/j.conengprac.2012.03.009

[20] Sauter, L., and Palmer, P., "Analytic Model Predictive Controller for Collision-Free Relative Motion Reconfiguration," Journal of Guidance, Control, and Dynamics, Vol. 35, No. 4, 2012, pp. 1069-1079. doi:10.2514/1.56521

[21] Wang, L., "Discrete Model Predictive Controller Design Using Laguerre Functions," Journal of Process Control, Vol. 14, No. 2, 2004, pp. 131-142. doi:10.1016/S0959-1524(03)00028-3

[22] Bemporad, A., Morari, M., Dua, V., and Pistikopoulos, E. N., "The Explicit Linear Quadratic Regulator for Constrained Systems," Automatica, Vol. 38, No. 1, 2002, pp. 3-20. doi:10.1016/S0005-1098(01)00174-1

[23] Wang, Y., and Boyd, S., "Fast Model Predictive Control Using Online Optimization," IEEE Transactions on Control Systems Technology, Vol. 18, No. 2, 2010, pp. 267-278. doi:10.1109/TCST.2009.2017934

[24] Lantoine, G., and Epenoy, R., "Quadratically Constrained LinearQuadratic Regulator Approach for Finite-Thrust Orbital Rendezvous," Journal of Guidance, Control, and Dynamics, Vol. 35, No. 6, 2012, pp. 1787-1797. doi: $10.2514 / 1.56961$

[25] Luo, Y. Z., Tang, G. J., and Lei, Y. J., "Optimal Multi-Objective Linearized Impulsive Rendezvous," Journal of Guidance, Control, and Dynamics, Vol. 30, No. 2, 2007, pp. 383-389. doi: $10.2514 / 1.21433$

[26] Clohessy, W. H., and Wiltshire, R. S., "Terminal Guidance System for Satellite Rendezvous," Journal of the Aerospace Sciences, Vol. 27, No. 9, 1960, pp. 653-658. doi: $10.2514 / 8.8704$

[27] Bando, M., and Ichikawa, A., "In-Plane Motion Control of HillClohessy-Wiltshire Equations by Single Input," Journal of Guidance, Control, and Dynamics, Vol. 36, No. 5, 2013, pp. 1-10. doi:10.2514/1.57197

[28] Richards, A., Schouwenaars, T., How, J. P., and Feron, E., "Spacecraft Trajectory Planning with Avoidance Constraints Using Mixed-Integer Linear Programming," Journal of Guidance, Control, and Dynamics, Vol. 25, No. 4, 2002, pp. 755-764. doi: $10.2514 / 2.4943$

[29] Pongvthithum, R., Veres, S. M., Gabriel, S., and Rogers, E., "Universal Adaptive Control of Satellite Formation Flying," International Journal of Control, Vol. 78, No. 1, 2005, pp. 45-52. doi:10.1080/00207170412331330887

[30] Bevilacqua, R., Romano, M., and Yakimenko, O., "Online Generation of Quasi-Optimal Spacecraft Rendezvous Trajectories," Acta Astronautica, Vol. 64, No. 2, 2009, pp. 345-358. doi: $10.1016 /$ j.actaastro.2008.08.001

[31] Mayne, D. Q., Rawlings, J. B., Rao, C. V., and Scokaert, P. O., "Constrained Model Predictive Control: Stability and Optimality," Automatica, Vol. 36, No. 6, 2000, pp. 789-814. doi:10.1016/S0005-1098(99)00214-9

[32] Rao, C. V., and Rawlings, J. B., "Linear Programming and Model Predictive Control," Journal of Process Control, Vol. 10, No. 2, 2000, pp. 283-289. doi:10.1016/S0959-1524(99)00034-7

[33] Shibata, M., and Ichikawa, A., "Orbital Rendezvous and Flyaround Based on Null Controllability with Vanishing Energy," Journal of Guidance, Control, and Dynamics, Vol. 30, No. 4, 2007, pp. 934-945. doi:10.2514/1.24171

[34] Valencia-Palomo, G., and Rossiter, J., "Using Laguerre Functions to Improve Efficiency of Multi-Parametric Predictive Control," Proceedings of the American Control Conference, IEEE, Piscataway, NJ, 2010, pp. 4731-4736. doi:10.1109/ACC.2010.5531098

[35] Coletti, M., Guarducci, F., and Gabriel, S., "A Micro PPT for Cubesat Application: Design and Preliminary Experimental Results," Acta Astronautica, Vol. 69, No. 3, 2011, pp. 200-208. doi:10.1016/j.actaastro.2011.03.008

[36] Busse, F. D., How, J. P., and Simpson, J., "Demonstration of Adaptive Extended Kalman Filter for Low Earth Orbit Formation Estimation Using CDGPS," Journal of the Institute of Navigation, Vol. 50, No. 2, 2003, pp. 79-93. 
[37] Woffinden, D. C., and Geller, D. K., "Relative Angles-Only Navigation and Pose Estimation for Autonomous Orbital Rendezvous," Journal of Guidance, Control, and Dynamics, Vol. 30, No. 5, 2007, pp. 14551469.

doi: $10.2514 / 1.28216$

[38] Vallado, D. A., Fundamental of Astrodynamics and Applications, 2nd ed., Microcosm Press, El Segundo, CA, 2001, pp. 511-524.

[39] Jacchia, L. G., "Revised Static Models of the Thermosphere and Exosphere with Empirical Temperature Profiles," SAO Special Rept. 332, SAO/NASA Astrophysics Data System, Harvard-Smithsonian Center for Astrophysics, Cambridge, MA, 1971, http://adsabs.harvard .edu/abs/1971SAOSR.332.....J [retrieved 23 July 2014].
[40] Starin, S. R., Yedavalli, R., and Sparks, A. G., "Spacecraft Formation Flying Maneuvers Using Linear Quadratic Regulation with No Radial Axis Inputs," AIAA Guidance, Navigation, and Control Conference and Exhibit, Montreal, AIAA Paper 2001-4029, Aug. 2001. doi:10.2514/6.2001-4029

[41] Yoon, H., and Agrawal, B. N., "Novel Expressions of Equations of Relative Motion and Control in Keplerian Orbits," Journal of Guidance, Control, and Dynamics, Vol. 32, No. 2, 2009, pp. 664-669. doi: $10.2514 / 1.38210$

[42] Vaddi, S. S., and Vadali, S. R., "Linear and Nonlinear Control Laws for Formation Flying," Advances in the Astronautical Sciences, Vol. 114, Pt. I, 2003, pp. 171-187, http://www.univelt.com/linkedfiles/ADV114 .html [retrieved 23 July 2014]. 